\title{
Integration of micro-optical elements with top-end of fibers via focused ion beam direct fabrication
}

\author{
Yongqi Fu*, Ngoi Kok Ann Bryan \\ * Innovation in Manufacturing Systems and Technology (IMST), Singapore-Massachusetts Institute of Technology \\ (MIT) Alliance, 50 Nanyang Avenue, Singapore 639798. (E-mail: yqfu@ntu.edu.sg) \\ School of Mechanical and Production Engineering, Nanyang Technological University, \\ 50 Nanyang Avenue, Singapore 639798
}

\begin{abstract}
Integration of micro-optical elements (MOEs) with optical fiber at top-end was realized by use of focused ion beam (FIB) direct milling. The designed MOEs (micro-refractive lens, microdiffractive lens with continuous relief, and micro-gratings) were directly milled by the FIB with ion energy and ion beam current of $40 \mathrm{keV}$ and $569 \mathrm{pA}$, respectively, in the core area of both single mode fiber and multimode finer for dispersion and focusing uses. The integrated fibers can be used in microspectrometer, optical fiber sensors, and micro-optical systems for biochemistry analysis.

Keywords: Diffraction gratings; Diffractive optics; Fiber design and fabrication; Microstructure fabrication; FIB
\end{abstract}

\section{Introduction}

Optical fiber is a key component for optical communication, optical sensor, and biochemistry analysis systems. With the tendency of integration and miniaturization, the optical systems become smaller and smaller, such as micro-optical electronic mechanical systems (MOEMS). However, R \& D of current micro-optical systems was limited by the problem of separate components in the systems, which is a bottleneck for the miniaturization of optical systems. M. Prasciolu reported a method of fabrication the micro-diffractive lens on-fiber by e-beam lithography. ${ }^{1}$ Eisenstein reported their conical lens on-fiber by use of chemically etching. ${ }^{2}$ In this paper, we focus on the point of integration of MOEs with optical fibers. The MOEs (micro-refractive lens, micro-diffractive lens with continuous relief, and micro-gratings) were directly integrated at core area of top-end of the fibers (both single mode and multimode fibers) by directly writing of the FIB. Before this, we studied the fabrication and integration of MOEs with edge-emitting laser diode and vertical cavity emission laser diode (VCSEL) by use of FIB technology with both functions of direct milling and deposition of $\mathrm{SiO}_{2}{ }^{3 \sim 9}$ These works show that the FIB technology is a practical way for the integration of MOEs with laser diodes for beam collimation and focusing. Our experiments were carried out with both single-mode and multimode fibers from corning company (SMF288.3/125, and MMF 50/125). A micro-grating was directly milled at core area of the single-mode fiber, and microdiffractive lens with continuous relief and micro-refractive lens were milled at core area of the multimode fibers, respectively, by the FIB milling.

\section{Experimental setup}

The milling experiments were carried out by our FIB machine (FEI Quanta 200 3D) with ion source of liquid gallium, integrated with scanning electron microscope (SEM). This machine uses a focused $\mathrm{Ga}^{+}$ion beam with energy of $5 \sim 30 \mathrm{keV}$, a probe current of $1 \mathrm{pA} \sim 20 \mathrm{nA}$ and beam limiting aperture size of $25 \mu \mathrm{m} \sim 350 \mu \mathrm{m}$. For the smallest beam currents, the beam can be focused down to $10 \mathrm{~nm}$ in diameter at full width and half maximum (FWHM). The ion beam is focused on the sample surface with normal incident angle. The ion beam scan in the defined area of $64.5 \times 64.5 \mu \mathrm{m}^{2}$ for the micro-grating and $75 \times 75 \mu \mathrm{m}^{2}$ for the diffractive lens, respectively, with the milling process parameters of ion dose, raster mode scanning frequency, pixel space, overlapping, and beam current, are $0.3 \mathrm{nC} / \mu^{2}, 2 \mathrm{~Hz}, 45 \mathrm{~nm}, 50 \%$, and $1 \mathrm{nA}$, respectively.

The diffractive structures can be milled by the FIB using its programming function. ${ }^{3-9}$ A computer program can be written (using the machine provided commands instead of the commonly used computer languages) in terms of the 
designed parameters and discrete data. Then run the program in a defined milling area. The manufacturing procedures are as follows:

- Discreting the relief curves according to selected ion beam spot size and feature size of the diffractive lenses.

- Selecting suitable ion energy (ion dose) and dwell time in terms of discrete relief height and curvature of the spherical lens so as to determine the total milling depth for each step (see Fig. 1 and Fig.2 (a) and (b)).

- Programming and optimizing the parameters.

- Milling the relief microstructures by running the designed computer programs.

- Measuring the accuracy of the relief profile and form by interferometers or atomic force microscopy (AFM) or optical interferometer, e.g. WYKO NT 2000.

- Revising machining parameters and recycling above procedures until fabrication accuracy of the diffractive lens reaches the design's.

The designed continuous profile of the micro-lens is divided into many thin layers with the circular shape slice with different diameters for the sake of fabrication, which compose of the lens 3 dimensional profiles using the discrete layers. Each layer is rectangular, with a different size for each different section.

The FIB also can fabricate the microlens on the top end of the fibers by deposition (additive material). ${ }^{[10]} \mathrm{SiO}_{2}$ can be deposited directly to form a required pattern by FIB deposition. The source gas is decomposed by an ion beam and adsorbed on the surface of the substrate. The entire process is controlled by a computer program, generally based on the relationship between ion-beam energy and deposited thickness. The whole process can be carried out automatically using the software program, as shown in Fig.3 and Fig.4. By changing the dwell time under different beam-limiting aperture sizes and controlling the actual beam current error to be less than $10 \%$ of the corresponding theoretical value, different deposition results were obtained with precursor gases of 1,3,5,7 tetramethylcyclotetrasiloxane (TMCTS) and $\mathrm{H}_{2} \mathrm{O}$ for the $\mathrm{SiO}_{2}$ microlens deposition. The accelerating voltage used was $50 \mathrm{kV}$, and the chamber base pressure was maintained at $9.1 \times 10^{-6}$ Torr during the whole deposition process.

The designed continuous profile of the micro-cylindrical lens is divided into many thin layers for the sake of fabrication, which compose of the lens 3 dimensional profile using the discrete layers. Each layer is rectangular, with a different size for each different section. The micro-cylindrical lens is composed of many deposited rectangular $\mathrm{SiO}_{2}$ films with different size and corresponding discrete thicknesses in terms of the sag of the lens, which overlap layer by layer. Finally, the microlens can be obtained by post-processing after completing the deposition of all layers, which are overlapped from bottom to top.

\section{Experimental results and discussion}

Two problems exist during the milling process: charging and polishing on the top of fiber. The former is caused by the accumulated positive ions on the insulate quartz of the fiber core and cladding. It deflected the beam during milling. Some FIB machine equipped with a part, called flood gun, to produce negative electrons to neutralize the positive ions. Unfortunately, our FIB machine (Quanta 200 3D) has no this part. To solve this problem, we wrapped the fiber with copper tape, see Fig.5 (a). The milling quality was improved greatly by this way. As we know, the specialized facility for polishing the top of fiber already appeared in some companies. But we have no this facility. In order to realize the polishing on top of fiber, we directly use FIB milling in the defined circular area with beam current as large as $20 \mathrm{nA}$. After $50 \mathrm{~min}$., the top of the fiber was well polished, as shown in Fig.5 (b).

Fig.6 (a) is the micro-grating with designed depth and period of $527.8 \mathrm{~nm}$ and $3.1 \mu \mathrm{m}$ fabricated by the FIB direct program controlled milling on the top of single mode fiber with core diameter of $8.2 \mu \mathrm{m}$. The integrated grating can be used as dispersion in micro-optical system. Fig.6 (b) is the micro-diffractive lens with four annulus fabricated by the FIB direct program controlled milling on the top of multi-mode fiber with core diameter of $50 \mu \mathrm{m}$. The integrated diffractive lens can realize directly focusing the emitting light from the fiber.

Unfortunately, our machine (Micrion 9500EX) breakdown now, and the new machine (Quatan 200 3D) has not equipped with the deposition of $\mathrm{SiO}_{2}$. We have no results can show at here. For more information regarding the lens deposition, can see Ref. [10].

\section{Summary}

In summary, the FIB direct milling and deposition processes can be used to locally fabricate microlenses on top of the optical fibers for both single mode and multimode. It can realize integrated of microlenses with optical fibers and other optical waveguides to avoid misalignment error existed in conventional optical systems with separate components. Hopefully, it can provide another options for research and development of micro-optical systems. 


\section{Acknowledgement}

This work was supported in part by the Funding for Strategic Research Program on Ultra-precision Engineering from the A*STAR (Agency for Science, Technology and Research, Singapore), and Innovation in Manufacturing Systems and Technology (IMST) Singapore-Massachusetts Institute of Technology (MIT) Alliance.

\section{References}

[1] M. Prasciolu, D. Cojoc, S. Cabrini, L. Businaro, P. Candeloro, M. Tormen, R. Kumar, C. Liberale, V. Degiorgio, A. Gerardino, G. Gigli, D. Pisignano, E. Di Fabrizio, R. Cingolani, "Design and fabrication of on-fiber diffractive elements for fiber-waveguide coupling by means of e-beam lithography." Microelectronic Engineering, 67-68, 169174 (2003).

[2] G. Eisenstein and D. Vitello, "Chemically etched conical microlenses for coupling single-mode lasers into single-mode fibers.” Applied Optics, 21(19), 3470-3474 (1982).

[3] Yongqi Fu, Ngoi Kok Ann Bryan, "Hybrid micro-diffractive-refractive optical element with continuous relief fabricated by focused ion beam for single-mode coupling”. Applied Optics, Vol.40, No.32, pp.5872-5876, 10 November 2001.

[4] Yongqi Fu, Ngoi Kok Ann Bryan, et.al, "Diffractive optical elements with continuous relief fabricated by focused ion beam for monomode fiber coupling”. Optics Express, Vol.7, No.3, pp.141-147, 31 July 2000.

[5] Yongqi Fu, Ngoi Kok Ann Bryan, "Investigation of hybrid microlens integration with vertical-cavity surfaceemitting lasers for free-space optical links". Optics Express, Vol.10, No.9, pp.413-418, May 2002.

[6] Yongqi Fu, Ngoi Kok Ann Bryan, "Investigation of direct milling of micro-optical elements with continuous relief on substrate by focused ion beam technology". Optical Engineering. 39 (11), pp. 3008 3013, November 2000.

[7] Yongqi Fu, Ngoi Kok Ann Bryan. "Investigation of diffractive/refractive microlens array fabricated by focused ion beam technology”. Optical Engineering. 40 (4), pp.511-516, April 2001.

[8] Yongqi Fu, Ngoi Kok Ann Bryan, et. Al, "Integrated micro-cylindrical lens with laser diode for single-mode fiber coupling”. IEEE Photonics Technology Letters. Vol.12, No.9, pp. 1213-1215, September 2000.

[9] Yongqi Fu, Ngoi Kok Ann Bryan, "Investigation of micro- diffractive lens with continuous relief with verticalcavity surface emitting lasers using focused ion beam direct milling”. IEEE Photonics Technology Letters. Vol.13, No.5, pp.424-426, May 2001.

[10] Yongqi Fu, Ngoi Kok Ann Bryan, Investigation of micromanufacturing micro-cylindrical lens by focused ion beam technology. Journal of Vacuum Sciences and Technology B Vol.19, No.4, pp.1259-1263, July/Aug. 2001. 


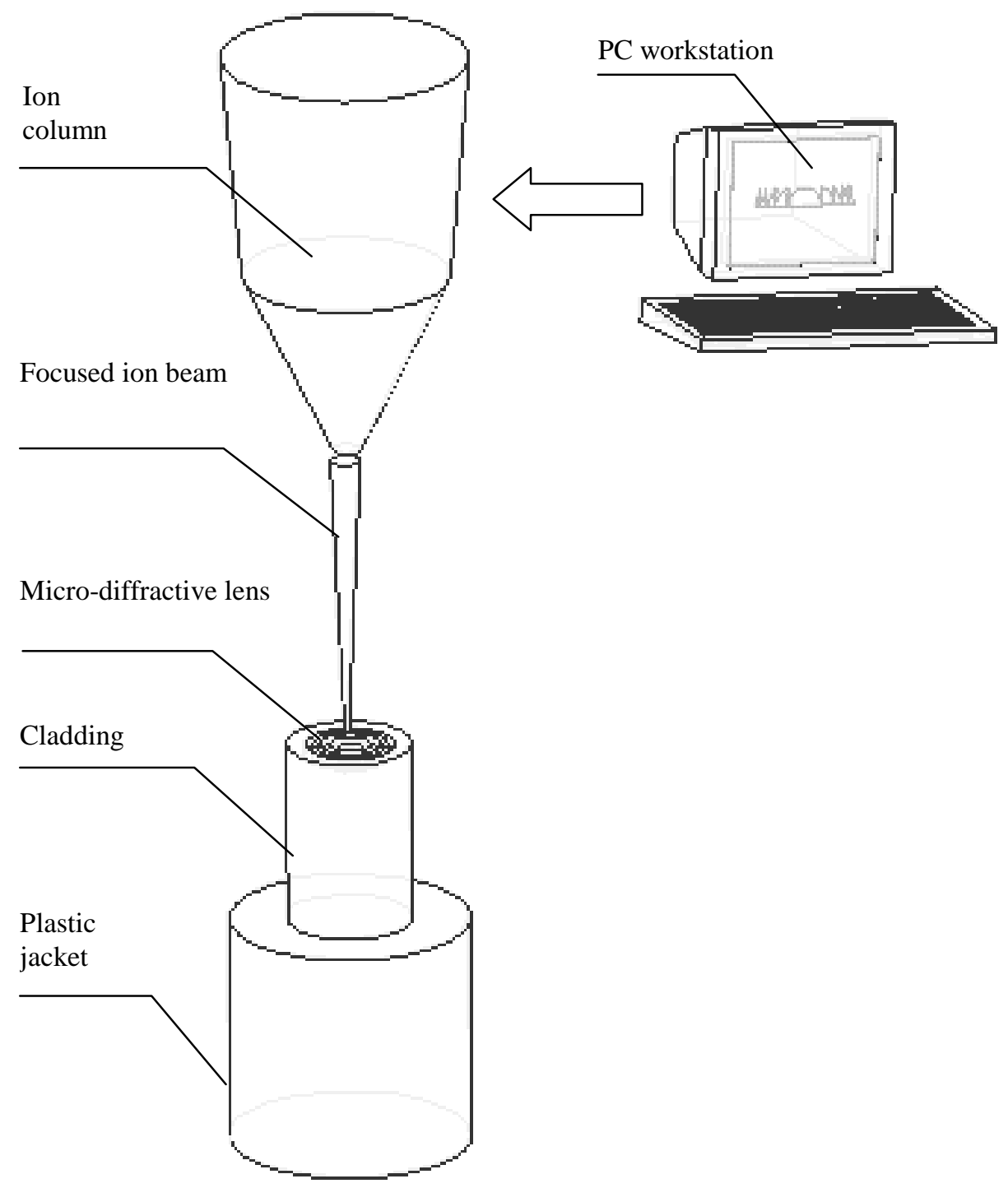

Fig.1 Schematic of micromachining of diffractive lens by computer program controlled focused ion beam 


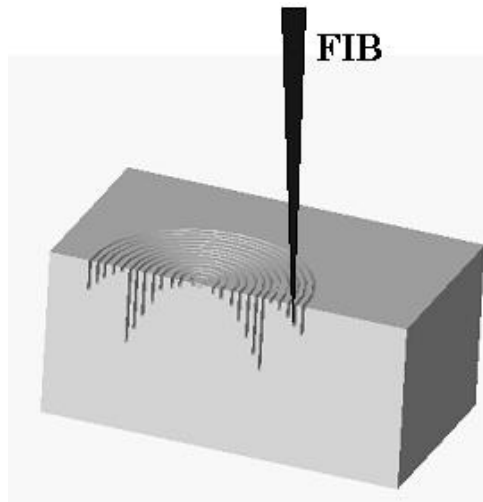

(a)

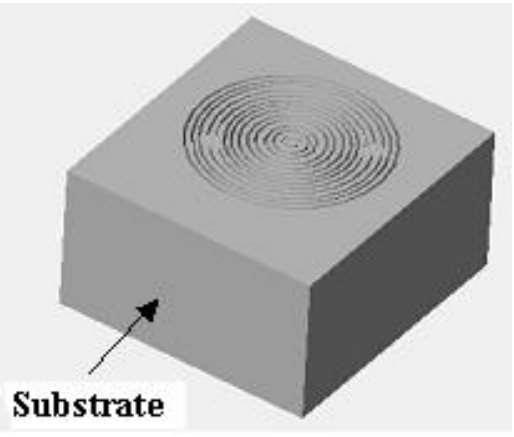

(b)

Fig.2 Schematic of programming controlled FIB milling for the diffractive structures with continuous relief. (a) cross-section of the FIB milling circleby-circle, different circles correspond to different milling depth; (b) top view of the Fresnel structure. It is the same principle for milling of blazed grating; only substitute the circles with straight lines.

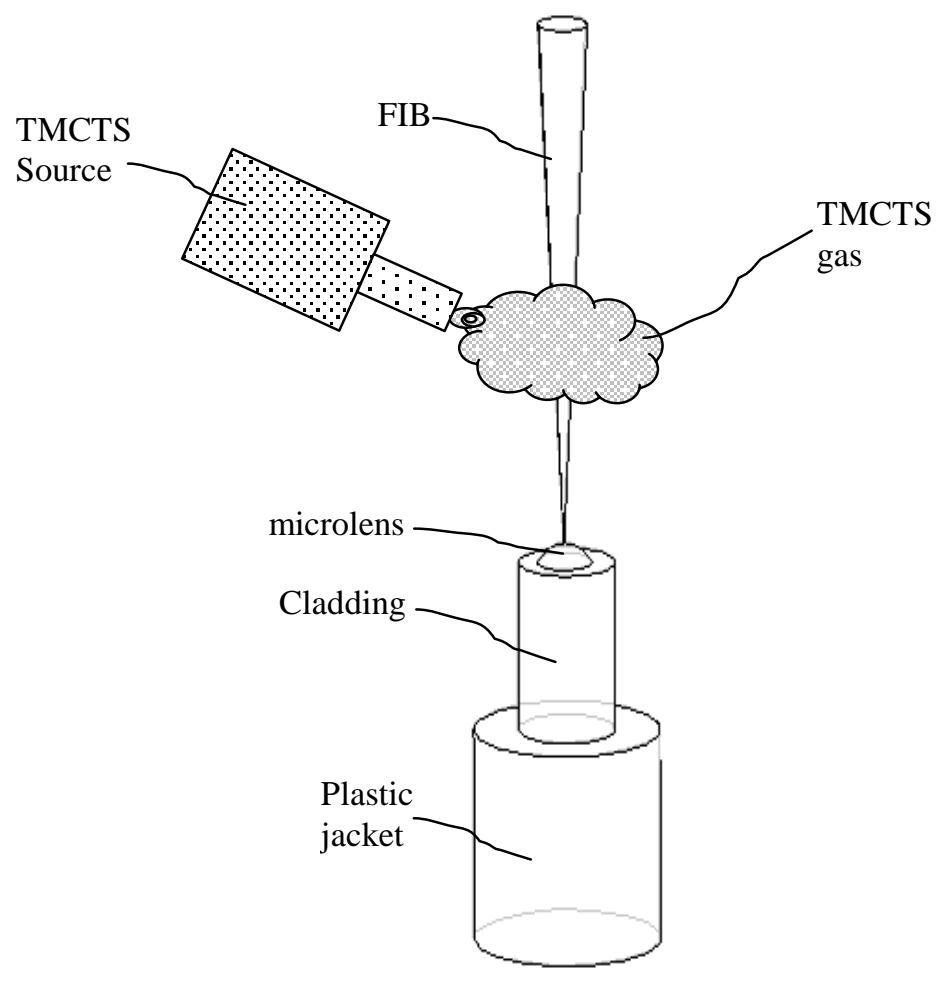

Fig.3 Schematic of deposition of microlenses by chemical gas of TMCTS via focused ion beam scanning. The whole process can be carried out by computer program or manual. 

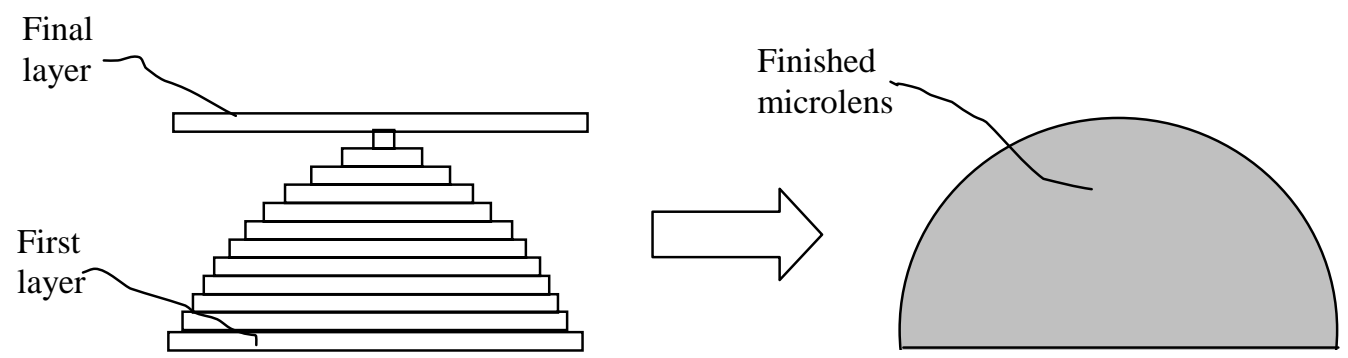

Fig.4 Schematic of microlens deposition through the method of layer-by-layer. Final layer was used for the post-processing.
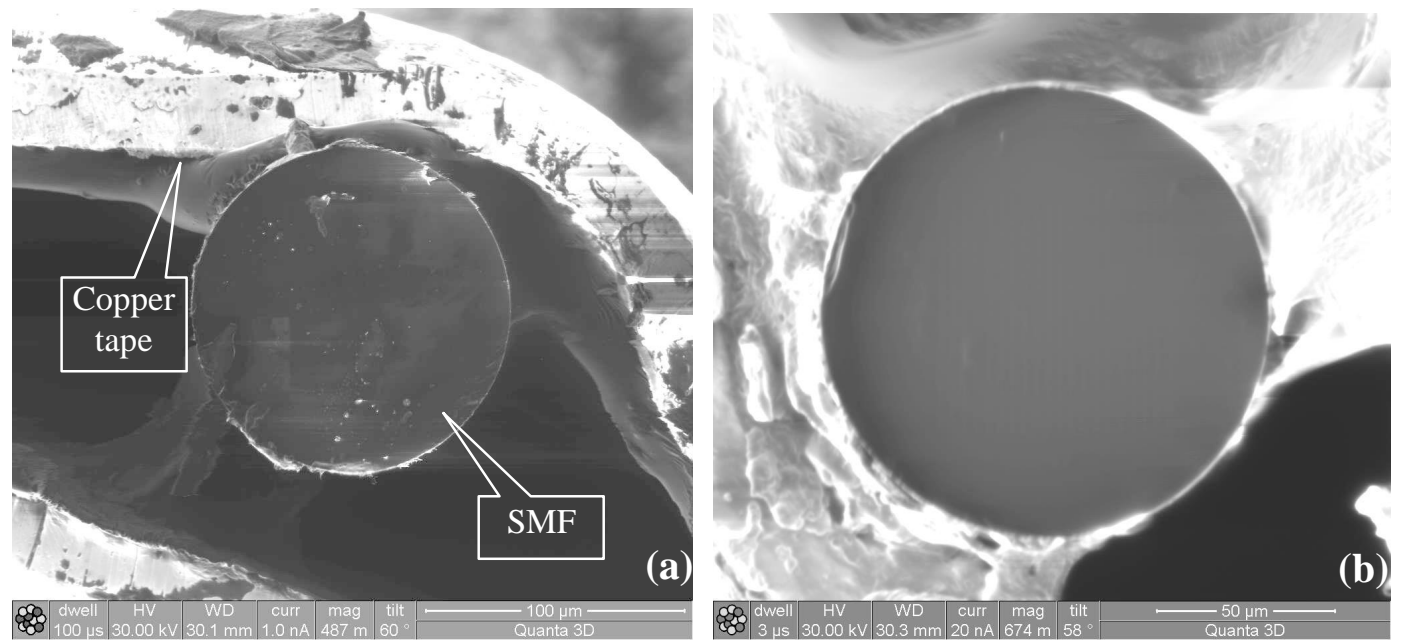

Fig.5 Results of FIB polishing on the top end of fiber. Micrograph of the fiber, (a) before FIB polishing; (b) after FIB polishing 

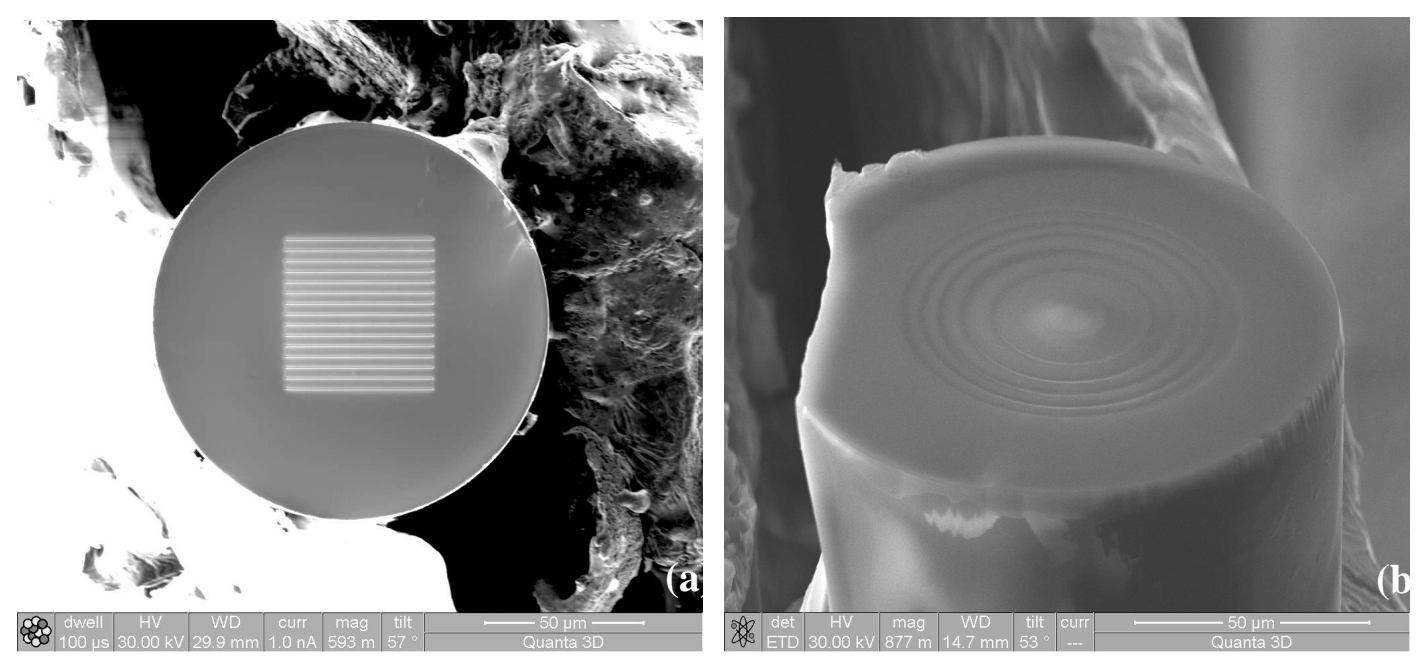

Fig.6 Results of fabrication of grating and diffractive lens on the top end of fiber. Micrograph of the fiber, (a) micro-grating by the FIB milling; (b) diffractive lens by the FIB milling without polishing before the fabrication. 